\title{
Picture Archiving and Communication System Bandwidth and Storage Requirements
}

\author{
Janice C. Honeyman, Walter Huda, Meryll M. Frost, Carole K. Palmer, and Edward V. Staab
}

\begin{abstract}
The purpose of this work was to determine the requirements for image storage and network bandwidth for a total digital department in a moderate sized academic radiology department. Data from the radiology information system was combined with image production information to produce a model of image acquisition. Destinations of images to reading rooms were studied to determine the final distributions of film. All findings were used to model the flow of data that would be expected if the images in the department were completely digital. Using today's standards, the department would produce approximately 15.7 Gbytes of data per day or 3.5 Tbytes of data per year if all acquisitions were digital. The peak acquisition rate would be 1.8 Gbytes per hour with a sustained rate greater than 1 Gbyte per hour for most of the working day. The anticipated bandwidth for the total digital department exceeded the capabilities of the existing picture archiving and communication system equipment. A distributed networked archive solution was shown to optimize access to images by radiologists and referring clinicians.
\end{abstract}

Copyright 01996 by W.B. Saunders Company

KEY WORDS: digital radiology, picture archiving and communication system (PACS), transmission images, display images, digitization images.

$\mathbf{P}$ ICTURE ARCHIVING and communication systems (PACS) offer radiology departments the potential for improved image data management efficiency. ${ }^{1-3}$ One major benefit of PACS is rapid and simultaneous dissemination of images to radiologist display stations and clinicians for review. ${ }^{4}$ Another important advantage is digital archival of images eliminating manual retrieval of old studies and thereby reducing problems of missing or lost films. ${ }^{5}$ PACS also offers prospects for direct interface with the radiology information system (RIS) that could integrate radiologist's reports and patient images. ${ }^{6,7}$

From the Department of Radiology, University of Florida, Gainesville; and Midwestern State University, Wichita Falls, $T X$.

Address reprint requests to Janice C. Honeyman, $P h D$, Director of Informatics, Department of Radiology, University of Florida, $1600 \mathrm{SW}$ Archer Rd, Box 100374, Gainesville, FL 32610-0374.

Copyright 1996 by W.B. Saunders Company

0897-1889/96/0902-0002\$3.00/0
A successful PACS implementation requires careful planning and analysis of data volumes generated, routed, displayed, and stored for future access. Previous estimates of the total amount of data generated by a radiology department with an annual volume of 100,000 examinations are on the order of 11 Gbytes per day. ${ }^{8}$ These daily acquisition rates correspond to annual image data generation of 2 to 3 Tbytes. However, designers of PACS networks will also need to take into account rates of flow of data and variations in the flow of data throughout the day for acquisition modalities, display stations, and image archives. ${ }^{2,9-13}$ It is important to specify a PACS network to handle the image transfer load using data that match the operation of the department for which PACS is being designed.

In this paper, we report on the pattern of image data generation and distribution from eight acquisition modalities to 11 diagnostic display locations. Data rates throughout the working day were calculated from RIS reports, and the contents of film folders. Results obtained provide quantitative information about the data storage requirements and anticipated data flow rates within our radiology department. These data were evaluated for their implications for prospective PACS network designs.

\section{MATERIALS AND METHODS}

Image generation. The University of Florida Radiology Department performs approximately 160,000 imaging examinations each year. Table 1 shows the department's annual imaging load grouped by similar image sources, the number of studies performed per year, and provides a summary of the major items of imaging equipment in the department. Examinations performed and interpreted in other departments, such as cardiac catherizations in cardiology, were excluded from this study.

Data were obtained on all examinations performed during a 4 consecutive week period. RIS provided the time and date of the performance of each radiographic study. Images of each image type were converted to megabytes using the factors summarized in Table 2. Choices for the values listed in Table 2 were made by considering the technical capabilities that are currently available and also estimating imaging requirements deemed necessary for radiological imag- 
Table 1. Eight Acquisition Clusters That Are Used to Perform Approximately 160,000 Imaging Examinations Each Year

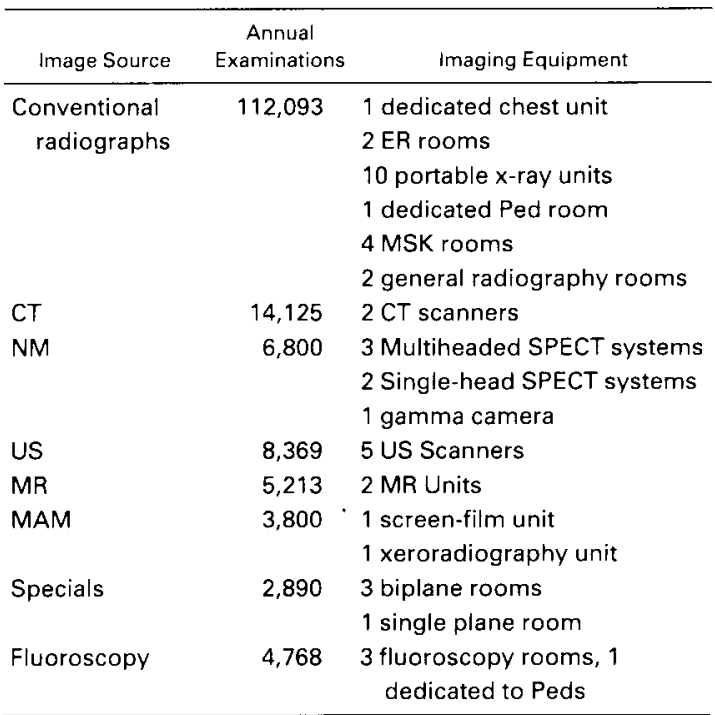

Abbreviations: SPECT, single-photon emission computed tomography.

ing. ${ }^{14,15}$ No account was taken of any patient "header" data or of image compression.

Technologists in computed tomography (CT), magnetic resonance imaging (MRI), and Special procedures maintain comprehensive records that include the date, the time, and the number of images produced for all examinations. All conventional radiographs were calculated at 10 Mbytes per film, regardless of the film size or the number of separate images recorded on the film. In conventional radiography,

Table 2. Image to Megabyte Conversion Factors

\begin{tabular}{|c|c|c|c|}
\hline $\begin{array}{l}\text { Image } \\
\text { Source }\end{array}$ & $\begin{array}{c}\text { Matrix } \\
\text { Size \& } \\
\text { Bytes/Pixel }\end{array}$ & $\begin{array}{l}\text { Mbytes } \\
\text { Per Image }\end{array}$ & Comments \\
\hline $\begin{array}{l}\text { Conventional } \\
\text { radiographs }\end{array}$ & $\begin{array}{l}2048 \times 2580 \\
2 \text { Bytes }\end{array}$ & 10 & Typical CR system \\
\hline CT & $\begin{array}{c}512^{2} \\
2 \text { Bytes }\end{array}$ & 0.5 & $\begin{array}{l}\text { Also applies to } \\
\text { scout views }\end{array}$ \\
\hline NM & $\begin{array}{l}32^{2}-512^{2} \\
2 \text { Bytes }\end{array}$ & $0.002-0.5$ & $\begin{array}{l}\text { Variable sized } \\
\text { data }\end{array}$ \\
\hline US & $\begin{array}{c}512^{2} \\
1 \text { Byte }\end{array}$ & 0.25 & $\begin{array}{l}\text { Video frame } \\
\text { grabbed }\end{array}$ \\
\hline MR & $\begin{array}{c}256^{2} \\
2 \text { Bytes }\end{array}$ & 0.125 & $\begin{array}{l}\text { Digital data from } \\
\text { scanner }\end{array}$ \\
\hline Mam & $\begin{array}{l}4096 \times 6144 \\
2 \text { Bytes }\end{array}$ & 48 & $\begin{array}{l}\text { Based on } 10 \\
\mathrm{lp} / \mathrm{mm} \text { resolu- } \\
\text { tion }\end{array}$ \\
\hline Specials & $\begin{array}{l}1024^{2} \\
2 \text { Bytes }\end{array}$ & 2 & Typical DSA value \\
\hline Fluoroscopy (I) & $\begin{array}{l}1024^{2} \\
1 \text { Byte }\end{array}$ & 1 & $\begin{array}{l}\text { Photo-spot } \\
\text { (Video-frame) }\end{array}$ \\
\hline Fluoroscopy (II) & $\begin{array}{l}2048 \times 2580 \\
2 \text { Bytes }\end{array}$ & 10 & Spot films (CR) \\
\hline
\end{tabular}

the majority of radiographic studies are performed using standard protocols with a specific number of images. Additional projections are recorded in the RIS. Mammography is a special type of digital radiography with high resolution requirements that are expected to result in an image size of 48 Mbytes.

In Fluoroscopy and Ultrasound (US), all film folders for the specified 4 week period were obtained and a record was made of the total number of films generated for each study. Photo-spot fluoroscopy films were calculated to require 1 Mbyte/image whereas spot films were calculated to be similar to CR with $10 \mathrm{Mbyte} /$ image. Digital US images are likely to be acquired through a video frame grabber which will result in .25 Mbyte/image. Digital archives in Nuclear Medicine (NM) provided both matrix size and number of images for each study. We reviewed the procedures performed during the 4 week period using the tape archives from NM to obtain the size of the studies and the number of images. Individual NM images vary from 0.002 to 0.5 Mbytes depending on the matrix size and the accurate numbers for each study were used to calculate the network and storage load.

RIS reports provided details of the acquisition locations of all studies in Radiology along with the time of the study. The rate of acquisition of image data as a function of time of day was generated for the "typical working day." Statistics for the typical working days were obtained by averaging the data generated from all the days in the study excluding weekends and holidays. Distributions of the sizes of total data generated per patient examination for each acquisition group, however, were obtained from the complete 4 week collection period of 28 days. The total number of studies for each acquisition group was taken from the RIS annual activity report. Annual image data (in megabytes) produced were obtained by multiplying the number of studies for each type of image by the mean examination data size.

Diagnostic display clusters. Images in the department are routed to display areas for diagnostic interpretation. The organ based display areas in radiology include Abdominal (Abd), Musculoskeletal (MSK), Specials-Visceral (Spc-V), Specials-Neuro (Spc-N), Chest, Mammography (Mam), Pediatrics (Ped), and Neuroradiology (Neuro). These sections, plus the Emergency Room (ER), US, and NM, correspond to 11 diagnostic display clusters where film or digital images are interpreted by radiologists.

Studies were assigned to diagnostic display clusters based on organ imaging sections or functional areas. Fluoroscopy, $\mathrm{CT}, \mathrm{MR}$, and digitized projection film studies were all distributed to one or more appropriate organ imaging sections. Mam, US, and NM are generally interpreted in the same location where they are generated. Some images were distributed to more than one diagnostic display cluster. For example, chest and abdomen CT studies were allocated to both the chest and abdomen diagnostic display clusters and studies originating in the ER were assigned to both the ER display cluster and to appropriate organ imaging sections. All images are also sent to the archive. We assumed for this research that sending images to multiple destinations does not require duplicate transmissions. 


\section{RESULTS}

Image acquisition. Table 3 is a summary of the image data acquisition in our radiology department. The stringent spatial resolution requirements of digital mammography result in the largest mean patient examination data size. The remaining data in Table 3 show that the largest amount of data generated is from digitized conventional radiographs. Conversely, NM, US, MR, and Special Procedures are least likely to cause problems for PACS network storage or bandwidth requirements.

Examination data size. Table 4 is a summary of the distribution of examination data sizes (megabytes) at each acquisition cluster. All four "digital" modalities (CT, NM, MR, and US) and Fluoroscopy show broad patient examination data size distributions, as do the distributions for Special Procedures. Conventional radiography and mammography showed well-defined peaks that correspond to the "representative" number of views for each examination under consideration (in this case these correspond to two views in conventional radiography and four views in mammography).

Three types of examinations have large median data sizes-Mam (192 Mbytes), Spc-N procedures (78 Mbytes), and Fluoroscopy (48 Mbytes). Most of the remaining categories of examinations have a range of data sizes from 8 to 16 Mbytes, except for NM (2.3 Mbytes) and CT (21 Mbytes). Individual studies for those examinations whose data size distributions have very long (upper level) tails, however, can significantly exceed these median values. Thus for

Table 3. Summary of Mean Patient Examination Data Sizes, Total Image Data Per Year, and the Acquisition Cluster Peak Image Data Acquisition Rate

\begin{tabular}{|c|c|c|c|}
\hline Sources & $\begin{array}{c}\text { Mean } \\
\text { Patient } \\
\text { Examination } \\
\text { Data Size } \\
\text { (Mbyte) }\end{array}$ & $\begin{array}{c}\text { Total Image } \\
\text { Data } \\
\text { Generated } \\
\text { Per Year } \\
\text { (Gbyte) }\end{array}$ & $\begin{array}{l}\text { Peak } \\
\text { Acquisition } \\
\text { Rate } \\
\text { (Mbyte/hr) }\end{array}$ \\
\hline Conventional radiographs & 21.3 & 2383 & 882 \\
\hline $\mathrm{CT}$ & 22.4 & 316 & 105 \\
\hline NM & 4.2 & 28 & 18 \\
\hline US & 6.9 & 58 & 38 \\
\hline MR & 13.2 & 68 & 27 \\
\hline MAM & 153.0 & 730 & 451 \\
\hline Spc-N & 122.0 & 73 & 63 \\
\hline Spc-V & 19.0 & 44 & 19 \\
\hline Fluoroscopy & 71.8 & 243 & 236 \\
\hline
\end{tabular}

Table 4. Data Size (Mbyte) per Study Distribution Percentiles

\begin{tabular}{llllll}
\hline \multirow{2}{*}{\multicolumn{1}{c}{ Image Source }} & \multicolumn{5}{c}{ Distribution \% } \\
\cline { 2 - 6 } & $10 \%$ & $25 \%$ & Median & $75 \%$ & $90 \%$ \\
\hline Conventional radiographs & 10 & 10 & 20 & 20 & 30 \\
CT & 10.5 & 12.5 & 21 & 27 & 38 \\
NM & 0.5 & 1 & 2.3 & 5.2 & 6.7 \\
US & 2.8 & 3.5 & 7.5 & 9 & 10.8 \\
MR & 7.4 & 9.6 & 12 & 15 & 21 \\
Mam & 115 & 192 & 192 & 192 & 192 \\
Spc-N & 48 & 48 & 78 & 120 & 240 \\
Spc-V & 2.4 & 8 & 8 & 24 & 48 \\
Fluoroscopy & 10.9 & 35 & 60 & 110 & 139 \\
\hline
\end{tabular}

examinations such as NM, Specials, and Fluoroscopy, significant numbers of studies will have considerably larger examination data sizes than their respective median values.

Total image traffic. Figure 1 shows the total rate of flow of image data that was obtained by summing all image acquisition data for a typical working day. The peak rate approaches 1.8 Gbytes/hour, and a rate exceeding 1 Gbyte/ hour is maintained throughout much of the working day. The total annual data generation is estimated to be 15.7 Gbytes/typical working day or 3.5 Tbytes/year if radiology was a total digital department. This does not take into consideration outside images that may be digitized for comparison or transmitted to our location for primary diagnostic interpretation.

Diagnostic display stations. Table 5 shows a detailed breakdown of the destinations of new patient images. Digitized conventional images, CT, MR, and Fluoroscopy studies are distributed to multiple diagnostic clusters. NM, US, Mam, and Special Procedure studies are gener-

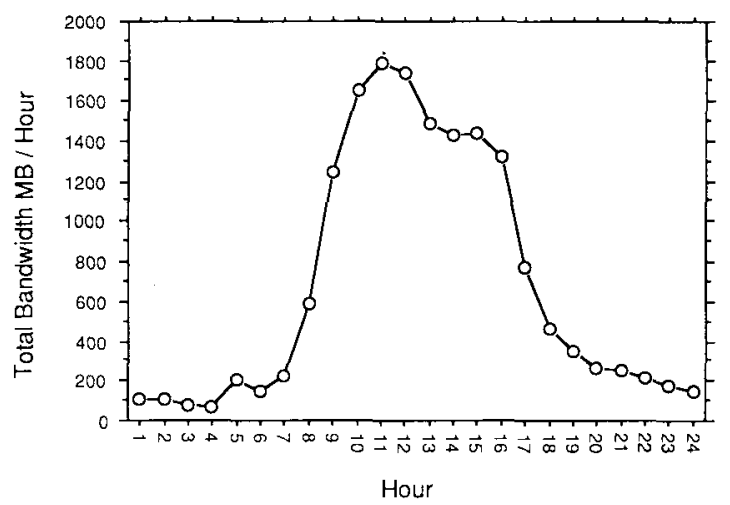

Fig 1. Total bandwidth in megabytes/hour anticipated if all the images in the radiology department were digital. 
Table 5. Distribution of Acquisition Cluster (Rows) Patient Data (Mbyte) to Diagnostic Display Clusters (Columns) for the "Typical Working Day"

\begin{tabular}{|c|c|c|c|c|c|c|c|c|c|c|c|}
\hline Cluster & Abd & MSK & Ped & NM & Spc-V & Spc-N & Neuro & Chest & Mam & US & ER \\
\hline Conv & 838 & 2,911 & 1,071 & & & & 213 & 2,406 & & & 1,330 \\
\hline CT & 365 & 129 & & & & & 561 & 181 & & & \\
\hline NM & & & & 118 & & & & & & & \\
\hline US & & & & & & & & & & 248 & \\
\hline MR & 18 & 91 & & & & & 145 & 5 & & & \\
\hline Mam & & & & & & & & & 3,352 & & \\
\hline Spc-N & & & & & & 178 & 57 & & & & \\
\hline Spc-V & & & & & 143 & & & & & & \\
\hline Fluoro & 1,163 & 34 & 311 & & & & & 4 & & & \\
\hline Total & 2,384 & 3,165 & 1,382 & 118 & 143 & 178 & 976 & 2,433 & 3,352 & 248 & 1,330 \\
\hline
\end{tabular}

The total data flow is 15.7 Gbytes/day.

ally routed to workstations associated with the modality. The diagnostic display clusters with the most challenging bandwidths are Mam, MSK, Chest, and Abd.

\section{DISCUSSION}

Subjective judgments are needed to describe the division of any radiology department into diagnostic display clusters. The clusters we defined closely matched the organ based imaging sections that are used in this department and which are similar to those currently encountered in many North American radiology departments. The exceptions were five functional areas, NM, ER, US, Spc-N and Spc-V, where acquired images are interpreted in reading rooms associated closely with the acquisition modalities. Our segmentation of the department into diagnostic display clusters is sufficiently precise to permit the generation of reliable examination data while permitting generalization of our quantitative findings to other radiology departments.

The specific values for examination data sizes, and the corresponding data flow rates, are directly related to the digitization factors assumed in our study (Table 2). The "digital" modalities (ie, CT, NM, and MR) have well established digital data formats that are likely to remain constant for the foreseeable future. For plain film radiography, the " $2 \mathrm{~K} \times 2.5 \mathrm{~K}$ pixel matrix" size used by many computed radiography (CR) systems was adopted given the deployment of CR in all currently designed "digital" radiology departments. ${ }^{16-19}$ Digital fluoroscopy was based on video frame capture of 1,000 line
TV systems similar to current digital subtraction angiography (DSA). In our department, all radiographic exposures during fluoroscopy make use of spot film (CR type) images except for pediatrics, which employs photospot film imaging. Virtually all mammographic imaging is currently performed with screen/film systems and the "digital" future of mammography is uncertain. Although high resolution CR (5 lp/ $\mathrm{mm}$ ) has been evaluated for mammographic screening, ${ }^{20-22}$ current digital detector designs envision a limiting spatial frequency of $10 \mathrm{lp} /$ $\mathrm{mm}$. $^{23}$

Using the data in Table 2, film images can be converted to their corresponding digital image size. Data presented in this study could be scaled to account for any differing digitization choices that may be achieved in the future, for example the high resolution $\mathrm{CR}$ plates that produce a $4 \mathrm{~K} \times 4 \mathrm{~K}$ pixel matrix. For any given cluster or archive, analysis of examination data should focus onto three key issues: (1) the total amount of data generated; (2) the rates at which these data are generated; (3) and the temporal variation in the acquisition rate. Total data generated should be used to specify archive and workstation storage requirements whereas data rates should be used to specify network bandwidth.

Just more than half of the diagnostic display clusters had data transfers rates in the range of 1 to 3.3 Gbytes/working day. In our experience, it has been desirable to keep studies for an average of 3 days on a workstation for convenient review. In our system, comparison studies retrieved from archives use approximately 50\% 
of the volume of new data on the workstations. Using the neuro-radiology workstation as an example, 933 Mbytes of daily data acquisition resuits in 4 Gbytes of local workstation storage requirements ( 2.8 Gbytes for 3 days new data and 1.4 Gbyte for comparison data). The anticipated rate at which primary patient image data would be transmitted to these diagnostic display clusters is in the range of 120 to 450 Mbytes/ hour.

A single archive in this department would need to cope with a peak image acquisition rate of 1.8 Gbytes per hour, and a total 24 hour acquisition of 15.7 Gbytes. Therefore it is clear that even if the only function performed by the archive was "storage" with no retrieval or distribution function, the requirements would push the limits of current technology. The total amount of data sent to an archive approaches 4 Tbytes/year, requiring high cost technology. One archive would have difficulty handling the anticipated workload without compromised throughput, and a single archive is a single point of failure. It is appropriate to investigate distributed archival capacity. Multiple archives are desirable in that storage and retrieval can be highly parallelized over several devices and subnets. This configuration would make catastrophic failure of the entire PACS system far less likely. In addition, it is possible to have a single "long-term" archive to store older images in a compressed format. To assure that the system is easy to use, a centralized data base would be needed to locate a study on the appropriate archive when needed and route it to the requesting display cluster. A concept similar to this has already been achieved in our department with separate NM and CT/MR archives. ${ }^{24}$

Figure $2 \mathrm{~A}$ to $\mathrm{D}$ shows the acquisition rate of distributed archives where several diagnostic display clusters "share" an intermediate ar-
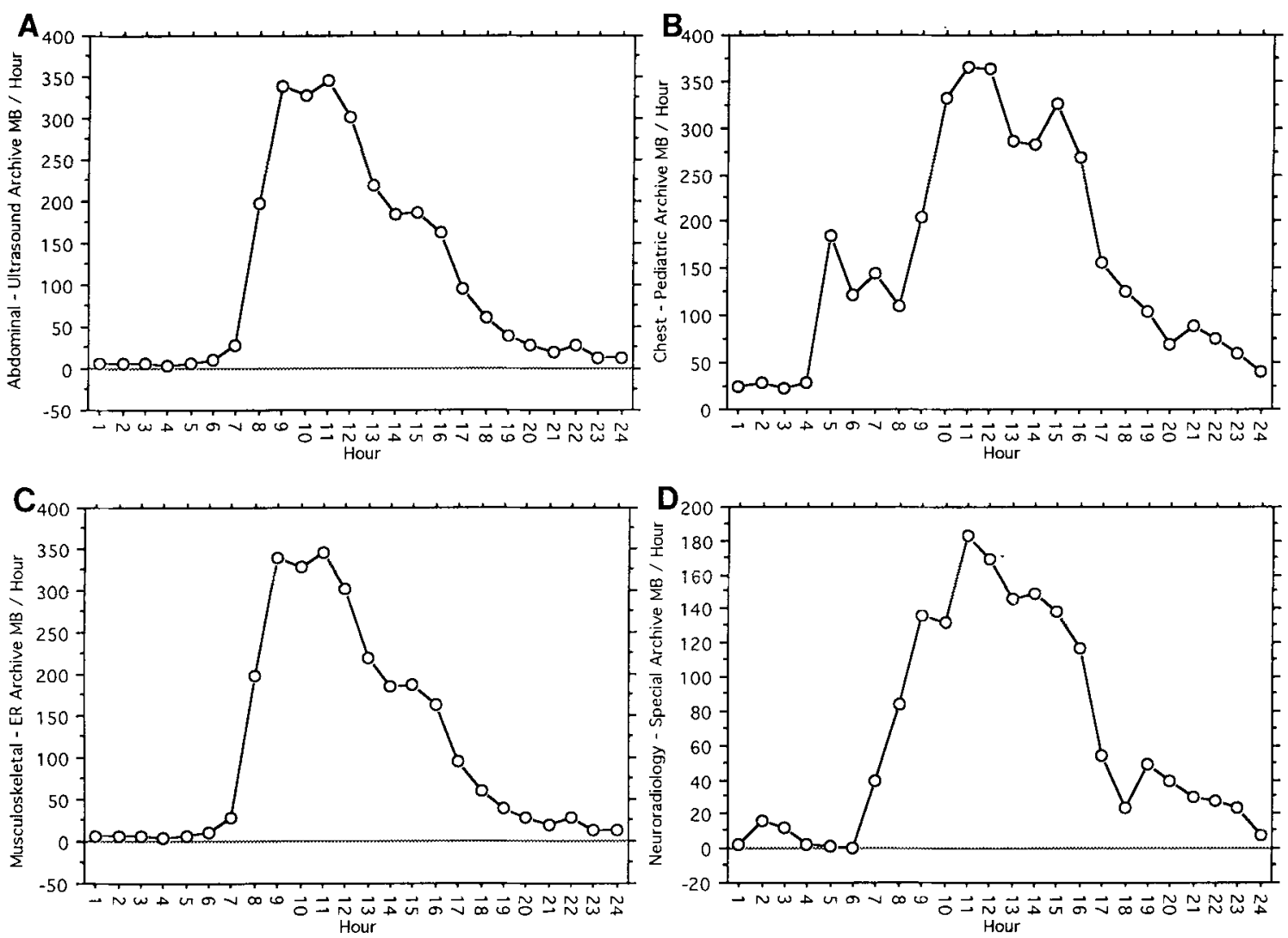

Fig 2. Total bandwidth to each of four intermediate archives anticipated if all the images in the radiology department were digital. (A) the Abd and US archive, (B) chest and Ped archive, (C) MSK and ER archive, and (D) Neuro and special procedures archive. 
chive. Display clusters were grouped according to likelihood of image sharing with Abd grouped with US, Chest grouped with Ped, MSK grouped with ER and Neuro grouped with all of Special Procedures. Mam and NM need their own archives, both because of special requirements. Mam has a very large data rate, but is expected to be among the last of the modalities to be implemented whereas NM data formats are difficult to integrate fully into suggested radiology standards. Sizes of distributed archives can be calculated from the collected data and can be adjusted to provide rapid access to newer studies whereas older studies can be stored on a single long-term archive.

Figure 3 describes the distributed archive design for the new PACS information management network currently being implemented to handle the volume anticipated by the results of the study. All Digital Imaging and Communications in Medicine acquisition computers have disk storage for approximately 2 days of images. In the case of network failure, technologists could continue to archive images for 2 days. The cluster storage devices shown on the right side of the page are intermediate term archives, each with 81 Gbytes of magnetic disk for rapid access to images. Each is designated for one or more workstation groups to reduce contention for disk and network resources. When possible, the intermediate archive is on the same side of a network bridge as the workstations it serves under normal operating conditions. For example the intermediate archive designated for

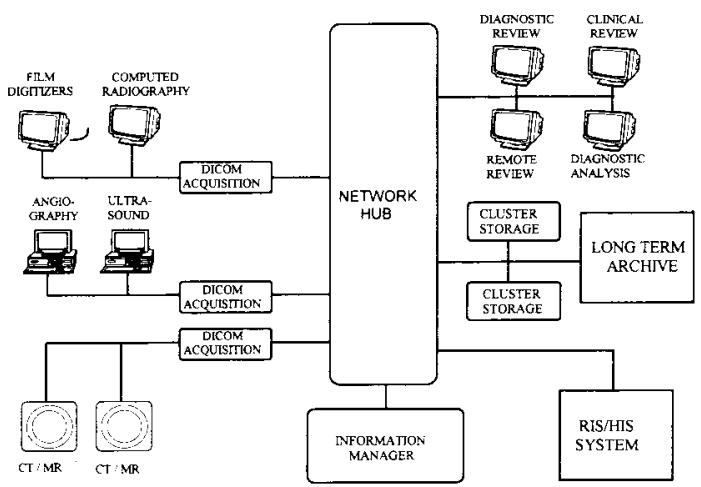

Fig 3. Design of the new PACS information management architecture to handle the bandwidth expected when all the images in the radiology department are digital. abdominal studies stores CT, MRI abdominal and chest studies as well as all US studies, and primarily serves workstations in the Abd, chest, and US reading rooms.

One very important factor that was omitted from the analysis is compression of image data. Current reversible compression algorithms are capable of achieving compression rates of about a factor of three. ${ }^{8}$ Irreversible compression is a controversial topic, but offers a potential compression factor of 15 to 20 and higher. It may be shown that higher (and irreversible) image compression ratios are possible without losing diagnostic image content. ${ }^{25}$ We chose to present uncompressed data to show a worst case scenario. Clearly, however, it would be straightforward to apply any selected compression option to our data, and modify the storage and transfer rates accordingly.

Two factors increase the storage and transfer requirements of all digital radiology departments. The first factor relates to the digitization, transfer, and storage of outside films. At referral teaching hospitals, many patients arrive with films obtained at other primary care diagnostic centers. If these films are digitized, transferred and stored, the amount of data is significant, and will further serve to increase the required storage and transfer capacities. In addition, with the new health care reform, alliances are being formed to provide expert reading of some studies at a central location through the use of teleradiology. Many institutions may be required to archive the digital images received from outside sources. The second factor relates to the need to send images to the referring physicians. For example, data may need to be transmitted to an intensive care unit to provide timely information obtained in portable films. ${ }^{26-28}$ Image information may also be sent to a referring clinic within an institution (eg, orthopedic clinic) who have referred a patient for a diagnostic examination, or to a more remote clinic as hospitals become more diversified into an ambulatory care environment in these days of health care. All these factors would serve to increase the bandwidth requirements and routing complexity of a PACS network. 


\section{REFERENCES}

1. Glass HI: Economic model of a whole hospital picture archiving and communication system installation. $J$ Digit Imaging 4:71-74, 1991 (suppl 1)

2. Honeyman JC, Messinger JM, Frost MM, et al: Evaluation of requirements and planning for picture archiving and communication systems. Radiographics 12:141-150, 1992

3. Honeyman JC, Frost MM, Huda W, et al: Picture Archiving and Communications Systems (PACS). Curr Probl Diagn Radiol XXIII:101-160, 1994

4. Bidgood WD, Staab EV: Understanding and using teleradiology. Semin Ultrasound CT MR 13:102-112, 1992

5. Worrell JA, Federspiel CF, Creasy JL, et al: Clinical impact of picture archiving and communication systems: Evaluation of a prototype system. J Digit Imaging 5:118-125, 1992

6. Levine B, Meissner M, Benson H, et al: Integration of a RIS with an IMACS. SPIE Med Imaging 1093:183-191, 1989

7. Mastodani J, Beeton C, Reed S, et al: Design of a multi-media communication system for clinical trials at the Ottawa Civic Hospital. SPIE Med Imaging 1093:230-239, 1989

8. Naylor AF: A strategy for the progressive introduction of picture archiving and communication systems for a radiology department. J Digit Imaging 5:82-88, 1992

9. Cox GG, Tempelton AW, Anderson WH, et al: Estimating digital information throughput rates for radiology networks: A model. Invest Radiol 21:162-166, 1986

10. Stewart BK, Taira RK, Dwyer SJ III, et al: Acquisition and analysis of throughput rates for an operational department-wide PACS. SPIE Med Imaging 1654:24-38, 1992

11. Wilson D, Smith D: Distribution of Workload Over the Working Day in a PACS. SPIE Med Imaging 2165:754762,1994

12. Wilson D, Glicksman R: Sizing a PACS. SPIE Med Imaging 2165:675-685, 1994

13. Huda W, Honeyman JC, Frost MM, et al: Networkbandwidth and archive-storage requirements model for PACS. SPIE Med Imaging 1899:14-2, 1993

14. Correia JA, Alpert NM: Properties of digital images in radiology. J Digit Imaging 1:18-23, 1988

15. Haynor DR, Smith DV, Park HW, et al: Hardware and software requirements for a picture archiving and communication system's diagnostic workstations. J Digit Imaging 5:107-117, 1992

16. Smith DV, Smith S, Bender GN, et al: Lessons Learned and Two Years Clinical Experience in Implementing the Medical Diagnostic Imaging Support (MDIS) System at Madigan Army Medical Center. SPIE Med Imaging $2165: 538-555,1994$
17. Siegel E: The Transition to the Filmless Imaging Department: Early Experience at the Baltimore VA Hospital, in Boehme JM, Rowberg AH, Wolfman NT, et al (eds): Computer Applications to Assist Radiology, vol 1. Carlsbad, CA, Symposia Foundation, 1994

18. Bryan S, Weatherburn G, Taylor J, et al: Evaluation of PACS at Hammersmith Hospital: Assessment of radiology department performance in the intensive care unit. SPIE Med Imaging 1899:434-440, 1993

19. Mosser H, Urban M, Partan G, et al: Clinical Routine Operation of a Filmless Radiology Department: 20 Months Experience, in Boehme JM, Rowberg AH, Wolfman NT et al (eds): Computer Applications to Assist Radiology, vol. 1. Carlsbad, CA, Symposia Foundation, 1994, pp 30-38

20. Oestmann JW, Kopans D, Hall DA, et al: A comparison of digitized storage phosphors and conventional mammography in the detection of malignant microcalcifications. Invest Radiol 23:725-728, 1988

21. Jarlman O, Samuelsson L, Braw M: Digital luminescence mammography. Early clinical experience. Acta Radiol 32:110-113, 1991

22. Higashida Y, Moribe N, Morita K, et al: Detection of subtle microcalcifications: Comparison of computed radiography and screen-film mammography. Radiology 183:483686, 1992

23. Yaffe MJ: Direct digital mammography using a scanned-slot CCD imaging system. Med-Prog-Technology 19:13-21, 1993

24. Honeyman JC, Frost MM, Staab EV, et al: Nuclear Medicine PACS with an Interfile to ACR-NEMA Interface and On-Line Medical Record. SPIE Med Imaging 1899:408412,1993

25. Goldberg MA, Pivovarov M, Mayo-Smith WW, et al: Application of Wavelet Compression to Digitized Radiographs, CT, and MR, in Boehme JM, Rowberg AH, Wolfman NT et al (eds): Computer Applications to Assist Radiology, vol 1. Carlsbad, CA, Symposium Foundation, 1994, pp 306-312

26. Gillespy T III, Staab EV, Lawrence E: Electronic Imaging in a Teaching Hospital Intensive Care Unit: Evaluation of the Clinical Review System. J Digit Imaging 2:124-128, 1990

27. Staab EV, Mitchell P, Williams J, et al: Teleradiology to the intensive care units at Shands Hospital. SPIE Med Imaging 1234:454-60, 1990

28. Staab EV, Honeyman JC, Frost MM, et al: Teleradiology in the local environment. SPIE Med Imaging 1446:1622, 1991 